\title{
Clinical applications of imaging modalities of the carpal joint in dogs with particular reference to the carpal canal
}

\author{
Angelika Tobolska, Zbigniew Adamiak, Joanna Głodek \\ Department of Surgery and Radiology, Faculty of Veterinary Medicine, \\ University of Warmia and Mazury in Olsztyn, 10-719 Olsztyn, Poland \\ angelika.tobolska@uwm.edu.pl
}

Received: June 24, 2019 Accepted: February 19, 2020

\begin{abstract}
The structure of the canine carpal joint is complex. This small joint consists of articulations that include the antebrachiocarpal, middle, carpometacarpal, and intercarpal joint surfaces. A large number of ligaments and tendons support and stabilise the carpus in dogs. Many injuries of this joint in dogs are not correctly recognised, diagnosed, or treated due to the limited use of diagnostic imaging methods. Radiography, the most common of them, has extensive application in diagnosing the causes of lameness in small animals. Other techniques, such as ultrasonography, computed tomography, and magnetic resonance imaging visualise other joint structures and surrounding soft tissues. However, these imaging modalities are rarely used to diagnose diseases and injuries of the canine carpus at present. The main reason for this is the small amount of research carried out and the lack of a properly described methodology for the use of imaging techniques. The wide use of all diagnostic imaging tools in the diagnosis of diseases and injuries of the wrist joint in humans shows that conducting studies on dogs could expand current knowledge. The use of these techniques in veterinary medicine could facilitate diagnosis and subsequent therapy of carpal disorders in dogs. MRI is the most frequently used imaging method in human medicine for visualisation of abnormalities of joints. This method could become a valuable part of the detection of inflammatory, traumatic, and degenerative diseases of the carpal joint in dogs.
\end{abstract}

Keywords: dog, carpus, CT, MRI, USG.

\section{Introduction}

The canine carpus is the compound joint formed by the carpal bones, carpal ligaments, and tendons as well as the region between the antebrachium and the metacarpus. The carpal bones of the dog are arranged in a proximal and a distal row. The elements of the proximal row are the intermedioradial, ulnar, and accessory carpal bones, and those of the distal row are the first, second, third, and fourth carpal bones. The carpal joints comprise antebrachiocarpal, middle, and carpometacarpal articulations. Between the individual carpal bones of each row are surfaces which are termed the intercarpal joints. On the palmar side of the carpus, the carpal canal is formed by the flexor retinaculum, the palmar carpal fibrocartilage, and the joint capsule. Ligamental stabilisation of the carpal joints in the dog is by the short medial and lateral collateral ligaments, dorsal and palmar radiocarpal ligaments, and ulnocarpal ligaments. Additionally, the accessory metacarpal ligaments and many short intercarpal ligaments connect the canine carpal bones transversely $(14,22,23,41)$.

The frequently seen injuries to the antebrachiocarpal joint are torn collateral ligaments, avulsions of ligaments from their attachments, or fractures of the styloid process. Acute luxation of the intermedioradial carpal bone is circumstantially seen. Open injuries are the results of subluxation of the antebrachiocarpal joint usually caused by road traffic accidents and are generally of the shearing type. Injuries to the middle carpal and carpometacarpal joints resulting in traumatic hyperextension are caused by falling from heights, jumping into deep excavations, and road traffic accidents. The symptom is that the patient walks on the palmar aspect of the carpus. The diagnosis of these injuries is often missed because hyperextended stressed 
radiographic views with the animal sedated are not taken immediately after the injury $(8,41,42)$.

Accessory carpal bone fracture is the most common in racing dogs. It also may be a consequence of a road traffic accident or a fall from a height. Fractures of the radial carpal bone, radial or ulnar styloid process, and the second or fifth metacarpal bones can also cause clinical signs in the carpal area. These traumas are less frequently seen than ligamentous injury. Traumatic injuries of the carpometacarpal joint are represented by luxation of one or more metacarpal bones. In puppies before ossification, epiphyseal separations of the accessory carpal bone, the distal radius, and ulna may be detected. Different less frequently diagnosed carpal disorders of dogs are distal growth plate defects of the radius or ulna, or both bones, congenital carpal defects, osteoarthrosis, and arthritis. Neoplasms of the carpal joint such as synovial sarcoma are uncommon. However, bone tumours generally appear in the distal radius and less often in the distal ulna $(8,41,42)$.

Carpal tunnel syndrome (CTS) and de Quervain syndrome are medical conditions that occur in humans. CTS is symptomatic compression neuropathy of the median nerve at the level of the wrist and has characteristic symptoms of hand pain, numbness, and tingling in the distribution of the median nerve, and reduction in grip strength and hand function. Pain and functional impairment of the hand are significant effects of carpal tunnel syndrome. It is typically diagnosed in patients between thirty and fifty years of age, and women are affected three times as often as men $(4,6)$. The present research showed that this syndrome is not diagnosed in dogs, but this animal could be used as a study model for median nerve compression in humans. Studies suggest that there are some similarities between dogs and humans when investigating some anatomic and physiological parameters: for example, the dimensions of the carpal canal and some ligaments $(37,38,39)$. There are many studies on the diagnosis and treatment of CTS in humans, and knowledge is constantly being improved $(4,6,12,20)$. Research carried out on canine carpal joints could help make progress towards better identification and reduction of human CTS. Racing and working dogs could suffer median nerve compression and display its symptoms, but CTS in dogs has not been diagnosed or described so far. The latency of the sensory nerve action potential of the median nerve in healthy dogs at forced hyperextension, hyperflexion, and in neutral positions of the carpus was recorded and described. This measurement was the shortest during hyperextension and prolonged during hyperflexion. It showed that increased compression on the median nerve could contribute to the progression of focal neuropathies (38). Tung et al. (37) conducted a comparative study to measure the compliance of the carpal tunnel in candidate animal models of CTS, by measuring the resistance when passing a tapered metal rod through the carpal tunnel. They showed that the compliance of the carpal tunnels in humans, dogs, rabbits, and rats are significantly different. Human carpal tunnels are the largest, and our transverse carpal ligaments are the thickest and longest. The features of the compliance for the dog carpal tunnel were closest and most similar mechanically to the human (37), suggesting that the dog might be a new model that could be used in more detailed study of CTS.

De Quervain syndrome in humans is the result of stenosing tenosynovitis of the first dorsal compartment of the wrist, which contains the tendons of the abductor pollicis longus and the extensor pollicis brevis. The main syndrome in patients is usually pain at the dorsolateral aspect of the wrist, which is related towards the thumb or lateral forearm or both (16). A similar syndrome has been described in dogs based on clinical and radiographic findings. Stenosing tenosynovitis of the abductor pollicis longus muscle in dogs revealed chronic lameness of the antebrachium and firm swelling at the medial aspect of the carpal joint (10).

Diagnostic imaging such as radiography, ultrasonography, computed tomography (CT), and magnetic resonance (MR) is an essential part of the diagnosis and treatment of lameness in small animals. Thanks to these methods, a veterinarian can confirm the suspected lesions, specify and document damage sites, assess the scope of and monitor a given disease, and suggest and plan surgical treatment. Imaging modalities are an essential part of the diagnosis of carpal disorders in dogs and humans. This article presents the use in practice of these techniques on canine patients. However, applications of imaging tools of carpal structures in dogs are not as well developed as in human medicine $(17,19,25,26,30,34,44)$.

\section{Radiography}

Radiological images can quickly and precisely provide morphological information about the localisation of the lameness after a physical examination (14). It is a quick method and the most common visualisation tool in diagnosis of carpal injuries. The standard views of the carpal joint are dorsopalmar and lateral. Stressed flexed, extended, and abducted views are required when the basic projections do not show disorders, for example, when an accessory bone fracture is suspected (41). The most common injuries detected on radiographs are fractures and dislocations of carpal bones. However, this imaging method can be appropriate for the diagnosis of primary bone tumours such as osteosarcoma. It is the most common bone tumour in dogs, and one of its locations in the forelimb is the distal radius, near to the carpal joint. Radiography can be useful in imaging carpal bone infections, most of which are of bacterial origin and are caused by direct inoculation, such as from an open injury, a bite wound, or surgery. Radiological images of canine carpal joints are also helpful for diagnosis of disorders such as decreased subchondral opacity, bone cysts, periarticular soft tissue swelling, joint displacement or incongruency, entheses and enthesophytes with abnormalities, and 
degenerative joint diseases. Examples of canine conformation disorders include valgus and varus deformities of the carpus (35), and for their diagnosis radiography is also highly suitable.

Radiological bilateral images are the basis of the diagnosis of intermedioradial carpal bone fractures in dogs. This injury is often manifested by chronic and intermittent lameness, carpal swelling, pain upon manipulation of the joint, and reduced range of motion. Research showed that sclerosis of the radial carpal bone adjacent to the fracture and carpal osteoarthritis could also occur (36). Avascular necrosis was described, which can appear with an intermedioradial carpal bone fracture in the dog. Radiographs delineated a focal periosteal reaction, soft tissue swelling, and an irregular intermedioradial carpal articular margin (28). Diagnosis of avascular necrosis of the intermedioradial carpal bone in dogs can be based on radiography, histopathology of the bone, and observed clinical signs. Indicative thereof is spotted osteolysis within the intermedioradial carpal bone, and it was visualised on dorsopalmar radiograph images. The studies suppose that this condition may be analogous to Preiser's disease, which is idiopathic avascular necrosis of the scaphoid bone of the wrist in humans (2).

Idiopathic ischaemic accessory carpal bone necrosis has also been reported in the dog. Radiography of the left carpus revealed expansile and lytic changes of the left accessory carpal bone, and additional results from a histologic evaluation of accessory carpal bone core biopsy specimens showed areas of bone necrosis. These findings were similar to previously reported findings for dogs with ischaemic femoral head necrosis and humans with ischaemic carpal pisiform or lunate bone necrosis (11). There has also been diagnosis of stenosing tenosynovitis of the abductor pollicis longus muscle in dogs according to radiographs of the carpus. Radiographic images showed a tubular bony proliferation distally to the radial groove, deeper radiolucent medial sulcus than in the opposite limb and soft tissue swelling medial to the carpus (13). A dorsomedial luxation of the radial carpal bone in the dog was also diagnosed by radiography and described. Radiographs of the carpus showed medial luxation of the radial carpal bone with its radial articular surface oriented medially and a tiny fracture of the fourth carpal bone on its latero-distal aspect (27).

Fractures of carpal bones and the distal radius are commonly diagnosed in human patients. Appropriate radiological evaluation of wrist fractures requires the experience and knowledge of an orthopaedic surgeon but may be insufficient to identify minor bone or ligament damage, and therefore other more advanced imaging methods have an essential role in the accurate diagnosis of wrist sprains and fractures in humans (9). Radiological diagnosis of wrist trauma in humans connecting with the navicular bone, radial styloid process, and first metacarpal fractures has been described. The recognition is based on displacement and obliteration of the navicular fat stripe on radiograms.
This useful radiographic feature is a small collection of fat located between ligaments and tendons of the human carpus (33). Radiological images are not an especially sensitive method for the evaluations of osteoarthritis of the wrist in humans. Assessment of carpal joints can be limited by overlapping structures because of the limb arrangement and projection (17).

\section{Ultrasonography}

Radiography is an essential method for assessing bones, but sonography can be useful to identify osteophytes surrounding soft tissue damage, inflammatory processes, and bone tumours. Ultrasonography examination of the musculoskeletal system in dogs is frequently performed using a 12-15 $\mathrm{MHz}$ linear transducer where the anatomical location permits. The examined limb should be raised while the patient is placed in the lateral position. Symptoms such as joint effusion, thickening, and rupture of the joint capsule can be recognised by ultrasonography. This examination can be an additional diagnostic method for differentiation of muscle or tendons injuries, instabilities of a joint, abscesses, foreign bodies, haematomas, soft tissue tumours, and tendinitis (15). Tendons and ligaments are made up of many closely aligned parallel fibres, and for that reason abnormalities are easily recognised as either high or low signal areas in the otherwise uniform structure. This imaging modality can also be used in evaluating the amount and character of synovial fluid and in localising periarticular mineralisation. Ultrasonography can be useful in accurate assessment of the periosteum, soft tissue tumours invading bone, and sequestration (14).

Research shows that ultrasonographic examination can be helpful in dogs to differentiate injuries of the tendon or tendon sheath of the abductor pollicis longus. This muscle fixes the carpus medially, and is an adductor of the canine carpal joint. Establishing fluid content, thickness of tendon, and the presence of mineralisation in the connective tissue is possible using ultrasonography (13). Studies have shown that tenosynovitis of the tendons of the extensor carpi radialis and common digital extensor muscles can be detected by ultrasound examination. This imaging method was an excellent complement to radiographs of the carpus which showed medial luxation of the intermedioradial carpal bone and a small fracture of the fourth carpal bone (27). The present studies showed the usefulness of ultrasonographic examination of the carpal canal in the dog. The median nerve can be seen as an ovoid shape with bright punctuate echoes during an ultrasound examination. It is also located on the posteromedial side of the median artery, which can be recognised by colour Doppler imaging (40).

Musculoskeletal ultrasonographic examination is used in human medicine during procedures such as arthrocentesis, tendon and articular injections, tissue biopsy, fluid aspiration, drainage catheter insertion, percutaneous tenotomy, and foreign body removal (30). 
In veterinary medicine for small animals, ultrasound examination can also be performed and be useful during the same procedures performed on joints (15) and is also used to detect joint disease abnormalities and to guide percutaneous procedures to treat conditions within the joints. It is a low-cost, non-invasive, effective, radiationfree imaging technique and a reasonable method to image superficial, wrist, and hand tendons and joints. Orlandi et al. (26) described ultrasound-guided procedures around wrist and hand structures such as the radiocarpal joint, small carpometacarpal joints, and tendons. In combination with power Doppler, this technology can also diagnose De Quervain's syndrome, a disease which is a stenosing tendosynovitis and tendonitis of the musculus abductor pollicis longus caused by a thickening of the retinaculum. These lesions hamper the normal sliding of the extensor pollicis brevis and the abductor pollicis longus tendons. Hypervascularity in the extensor compartment and retinaculum thickening can be seen on ultrasound images, which were reported as having guided procedures for diagnosing and administering injections to treat de Quervain's syndrome (26). This condition was also described in dogs, and it is termed stenosing tenosynovitis of the abductor pollicis longus muscle. It is manifested by chronic lameness of the forelimb and pain in hyperflexion of the carpus with a swelling at the medial aspect of the carpal joint (10).

\section{Computed tomography}

Computed tomography (CT) imaging allows high contrast and resolutions of osseous tissues by eliminating opacities caused by superimposed tissues. Therefore, a more accurate view of bone contours and articular surfaces is gained with CT images. CT provides better three-dimensionality of images by the tomographic display of CT slices than images have which are obtained with conventional radiography. A CT scan can detect osteolysis and osteogenesis earlier than standard imaging techniques. Another useful benefit of this method is the ability to view images in several image planes, which can help to define fracture localisation or bone fragmentation better. CT has also been used in the diagnosis of osteochondral defects and other bone growth diseases. Like all imaging methods, CT also has disadvantages such as artefacts caused by metal devices and orthopaedic implants, and tomography slice thickness (14).

Research has shown that for most of the anatomic structures evident in tissue sections, corresponding CT and MR imaging features were identified in the manus in canine cadavers. Osseous and musculotendinous structures of the manus were readily detected in CT and MR images (24). CT image-based measurements of the canine carpal canal were taken to establish the standard size and shape. It was shown that dimensions of the canine carpal canal were wider in the distal than the proximal part. Therefore, it could be suggested that the anatomical and morphometric structures of the carpal canal in dogs resemble those in humans. The authors of the study also presented the possibility of the occurrence a focal neuropathy called carpal tunnel syndrome in dogs analogous to this disease in people (39). CT may be a functional tool in the diagnosis of and planning of corrective surgery for forelimb deformities in dogs (7).

In human medicine, the use of high-resolution CT in wrist ligament injuries was evaluated and emerged as a method revealing the site of rips or perforations with greater precision than conventional arthrography (34). However, research showed that high resolution CT of the wrist is not a useful method for preoperative evaluation of human patients with CTS. Carpal tunnel measurements obtained by CT showed no significant difference between patients with clinical symptoms and the control group (20).

\section{Magnetic resonance imaging}

Magnetic resonance imaging (MRI) is used to detect musculoskeletal disorders of canine pelvic and thoracic limbs. Examination commonly includes the shoulder, elbow, carpal, stifle, and tarsal joints. MRI can be helpful when radiographs are not sufficient to make the ultimate diagnosis because of its ability to show the condition of soft tissues and abnormal fluid content. A patient is positioned for MRI examination under general anaesthesia, needing to be placed in lateral recumbency for imaging of the carpal joint with the lowered limb on the surface coil and the forelimb and carpal joint in a neutral, straight position. Rupture of the accessory metacarpal ligaments and the associated soft tissue trauma are caused by hyperextension subluxation injuries and can be identified on MRI. This imaging modality can also detect joint effusion, peritendinous fluid accumulation, foreign bodies, and osteomyelitis $(1,29,31)$. MRI is typically performed in several different acquisition sequences that emphasise different magnetic properties of tissues; commonly T1- and T2weighted and proton density (PD) properties are illustrated. The advantages of MRI in orthopaedic disease are the ability to acquire images in any desired anatomic plane and select the slice orientation relative to specific structures, which confer greater diagnostic capability on this modality. MRI can also render the contrast very clearly between osseous and soft tissue structures $(1,15)$. T1-weighted spin-echo images are preferred in imaging of articular cartilage because of their excellent depiction of anatomic detail and the high contrast between cartilage and subchondral bone. T2-weighted spin-echo images are also useful because of the visualised high signal intensity of joint fluid and the detection of internal changes in signal intensity within the articular cartilage itself. MRI could be useful in the assessment of the intensity of inflammatory changes within the subchondral bone and extent of subchondral lesions in dogs with osteochondritis lesions (5). The primary purpose of imaging the carpal joint is the examination of traumas caused by 
hyperextension injuries or rupture of the accessory carpal ligament. Images with high short tau inversion recovery or fat-suppressed T2 or PD signals are required to visualise peritendinous fluid accumulation and joint effusion and sagittal and transverse T1-weighted and T2-weighted images will reveal rupture of the accessory carpal ligament (31).

Previous research showed that all canine carpal ligaments plus the radioulnar articular disc and the palmar fibrocartilage were identified on MRI in the dorsal and sagittal planes. Anatomical structures in histological sections correlated with MRI (23). MRI is useful in diagnosing carpal avascular necrosis noninvasively and may allow an earlier diagnosis than with standard radiographs. Bone fractures can be an effect of avascular necrosis, and those of the intermedioradial carpal bone are good examples (28). However, studies conducted on canine patients showed that the MRI signal can be normal in necrotic avascular radial carpal bones. In most cases, the signal did not correlate directly with bone blood flow in an early stage of the avascular necrosis of the bone, although in one case all revascularised radial carpal bones showed decreased $\mathrm{T} 1$ signals and increased T2 signals (43).

MRI is an essential diagnostic tool in cases of chronic wrist pain in human patients, so much so that it has become the recommended method in the assessment of carpal osteoarthritis. MRI is better at revealing articular cartilage wastage and osteoarthritis changes than radiographs $(17,44)$. The evaluation of wrist ligament integrity typically focuses on three ligamentous complexes: radiologists and clinicians in human medicine mostly assess the scapholunate ligament, lunotriquetral ligament, and the triangular fibrocartilage complex on MRI scans. However, intrinsic, volar, and dorsal extrinsic ligaments of the wrist and ligaments of the thumb can be delineated during MRI examinations with improved techniques. Diagnosis of scaphoid dorsal subluxation on a sagittal MRI of the wrist may be an essential marker for scapholunate ligament tears $(3,18)$. The normal anatomy and related disorders of dorsal extensor tendons of the wrist could also be made visible using MRI. Research has produced the conclusion that MRI is an essential imaging modality for diagnosis of the cause of carpal pain associated with extensor tendons injuries in humans (19). In particular, high-field MRI can be of essential value in recognising cartilage damage in the distal carpal row and evaluating intrinsic ligaments injury in human medicine (25). Research conducted on paediatric patients demonstrated that MRI of the wrist significantly changes the clinical diagnosis in the majority of cases. Furthermore, the reason for changing the management of a condition in a substantial proportion of children was found to be an MRI examination (32). Low-field MRI of the carpal tunnel in humans provides a clear delineation of its soft tissue contents and bony boundaries which is beneficial in MRI scans of the wrists of patients with symptoms of CTS because they can give additional information about the condition of the soft tissues and median nerve (12).

\section{Conclusion}

Many conditions of the carpal joint causing thoracic limb lameness in dogs can be diagnosed using imaging techniques. Changes in bone structure can be observed not only on the X-ray image but also on images obtained by ultrasound, MRI, and CT. These methods also, to a different degree, show damage to other joint structures such as ligaments, tendons, and the joint capsule, and synovial fluid abnormalities $(5,13,14,15$, 31). The more precise imaging techniques are rarely used in veterinary practice due to the small size of carpal joint structures in dogs and high costs of the examination. The small number of examinations performed on these animals do not allow the usefulness of various visualisation diagnostic tools to be assessed. Advances in human medicine and the results of some experiments conducted on dogs show prospects for the more frequent use of ultrasound, computed tomography, and magnetic resonance imaging. MRI is the preferred imaging modality for diagnosis of traumatic, degenerative, and inflammatory diseases of joints in humans $(12,18,44)$ and is also recommended for diagnosing pathological changes of joints and determining treatment and prognosis of recovery in horses. Chronic lameness caused by navicular syndrome in horses is evaluated by magnetic resonance with great success (21). It is necessary to determine if abnormalities can be detected in canine carpal ligaments and other surrounding soft tissues using MRI, because it would allow for a more precise diagnosis and reduce the cost of treatment. If MRI examination was useful in the imaging of wrist joints in a group of children, it should be possible to demonstrate the effectiveness of this technique also in the case of dogs, by virtue of the greater closeness of the sizes of the child and canine joints than the adult and canine.

However, a greater amount of research on the methods of carpal imaging in dogs would not only allow human diseases to be researched. Proper interpretation of images enables more accurate diagnosis and appropriate therapy in veterinary medicine as well. Disorders of the carpal joint in dogs, which are difficult to diagnose by conventional methods, need the use of ultrasonography, MRI or CT for them to be confidently recognised $(14,15)$. Therefore, imaging technique methodology development is required for the diagnosis of these diseases of the carpal area in dogs.

Conflict of Interests Statement: The authors declare that there is no conflict of interests regarding the publication of this article.

Financial Disclosure Statement: Statutory funds of the Department of Surgery and Radiology, Faculty of 
Veterinary Medicine, University of Warmia and Mazury in Olsztyn.

Animal Rights Statement: None required.

\section{References}

1. Adamiak Z., Jaskolska M., Matyjasik H., Pomianowski A., Kwiatkowska M.: Magnetic resonance imaging of selected limb joints in dogs. Pol J Vet Sci 2008, 14, 501-505.

2. Aiken M.J., Stewart J.E., Anderson A.A.: Avascular necrosis of the canine radial carpal bone: a condition analogous to Preiser' $\mathrm{s}$ disease? J Small Anim Pract 2013, 54, 374-376.

3. Bateni C.P., Bartolotta R.J., Richardson M.L., Mulcahy H., Allan C.H.: Imaging key wrist ligaments: what the surgeon needs the radiologist to know. Am J Roentgenol 2013, 200, 1089-1095.

4. Burton C., Chesterton L.S., Davenport G.: Diagnosing and managing carpal tunnel syndrome in primary care. Br J Gen Pract 2014, 64, 262-263.

5. Carrig C.B.: Diagnostic imaging of osteoarthritis. Vet Clin North Am Small Anim Pract 1997, 4, 777-814.

6. D'arcy C.A., McGee S.: Does this patient have carpal tunnel syndrome? J Am Vet Med Assoc 2000, 283, 3110-3117.

7. Dismukes D.I., Fox D.B., Tomlinson J.L., Essman S.C.: Use of radiographic measures and three-dimensional computed tomographic imaging in surgical correction of an antebrachial deformity in a dog. J Am Vet Med Assoc 2008, 232, 68-73.

8. Earley T.D., Dee J.F.: Trauma to the carpus, tarsus, and phalanges of dogs and cats. Vet Clin North Am Small Anim Pract 1980, 10, $717-747$.

9. Goldfarb C.A., Yin Y., Gilula L.A., Fisher A.J., Boyer M.I.: Wrist fractures: what the clinician wants to know. Radiology 2001, 219, $11-28$.

10. Grundmann S., Montavon P.M.: Stenosing tenosynovitis of the abductor pollicis longus muscle in dogs. Vet Comp Orthop Traumatol 2001, 14, 95-100.

11. Harris K.P., Langley-Hobbs S.J.: Idiopathic ischemic necrosis of an accessory carpal bone in a dog. J Am Vet Med Assoc 2013, 12, 1746-1750.

12. Healy C., Watson J.D., Longstaff A., Campbell M.J.: Magnetic resonance imaging of the carpal tunnel. J Hand Surg Eur 1990, 15, 243-248.

13. Hittmair K.M., Groessl V., Mayrhofer E.: Radiographic and ultrasonographic diagnosis of stenosing tenosynovitis of the abductor pollicis longus muscle in dogs. Vet Radiol Ultrasound 2012, 53, 135-140.

14. Hoskinson J.J., Tucker R.L.: Diagnostic imaging of lameness in small animals. Vet Clin North Am Small Anim Pract 2001, 1, 165-180.

15. Kramer M., Gerwing M., Hach V., Schimke E.: Sonography of the musculoskeletal system in dogs and cats. Vet Radiol Ultrasound 1997, 38, 139-149.

16. Kulthanan T., Chareonwat B.: Variations in abductor pollicis longus and extensor pollicis brevis tendons in the Quervain syndrome: a surgical and anatomical study. Scand J Plast Reconstr Surg Hand Surg 2007, 41, 36-38.

17. Li A.E., Lee S.K., Rancy S.K., Burge A.J., Potter H.G., Wolfe S.W.: Comparison of magnetic resonance imaging and radiographs for evaluation of carpal osteoarthritis. J Wrist Surg 2017, 6, 120-125.

18. Meister D.W., Hearns K.A., Carlson M.G.: Dorsal scaphoid subluxation on sagittal magnetic resonance imaging as a marker for scapholunate ligament tear. J Hand Surg Am 2017, 42, 717-721.

19. Meraj S., Gyftopoulos S., Nellans K., Walz D., Brown M.S.: MRI of the extensor tendons of the wrist. AJR Am J Roentgenol 2017, 209, 1093-1102.

20. Merhar G.L., Clark R.A., Schneider H.J., Stern P.J.: Highresolution computed tomography of the wrist in patients with carpal tunnel syndrome. Skeletal Radiol 1986, 15, 549-552.
21. Mieszkowska M., Adamiak Z., Mieszkowski M., Holak P., Wolińska K.: Magnetic resonance imaging provides a detailed perspective on the navicular syndrome in horses. Med Weter 2016 ,72, 298-302.

22. Mikic Z.D., Ercegan G., Somer T.: Detailed anatomy of the antebrachiocarpal joint in dogs. Anat Rec 1992, 233, 329-334.

23. Nordberg C.C., Johnson K.A.: Magnetic resonance imaging of normal canine carpal ligaments. Vet Radiol Ultrasound 1999, 2, $128-136$.

24. Ober C.P., Freeman L.E.: Computed tomographic, magnetic resonance imaging, and cross-sectional anatomic features of the manus in cadavers of dogs without forelimb disease. Am J Vet Res 2009, 12, 1450-1458.

25. Ochman S., Wieskotter B., Langer M., Vieth V., Raschke M.J., Stehling C.: High-resolution MRI (3T-MRI) in diagnosis of wrist pain: is diagnostic arthroscopy still necessary? Arch Orthop Trauma Surg 2017, 137, 1443-1450.

26. Orlandi D., Corazza A., Silvestri E., Serafini G., Savarino E.V., Garlaschi G., Mauri G., Cimmino M.A., Sconfienza L.M.: Ultrasound-guided procedures around the wrist and hand: how to do. Eur J Radiol 2014, 83, 1231-1238.

27. Palierne S., Delbeke C., Asimus E., Meynaud-Collard P., Mathon D., Zahra A., Autefage A.: A case of dorso-medial luxation of the radial carpal bone in a dog. Vet Comp Orthop Traumatol 2008, 21, 171-176.

28. Pownder S.L., Cooley S., Hayashi K., Bezuidenhout A., Koff M.F., Potter H.G.: Non-invasive magnetic resonance imaging diagnosis of presumed intermedioradial carpal bone avascular necrosis in the dog. Can Vet J 2016, 8, 879-881.

29. Przeworski A., Adamiak Z., Glodek J.: Comparison of high-field and low-field magnetic resonance imaging of stifle joint disorders in dogs. Pol J Vet Sci 2016, 19, 663-670.

30. Royall N.A., Farrin E., Bahner D.P., Stawicki S.P.: Ultrasoundassisted musculoskeletal procedures: a practical overview of current literature. World J Orthop 2011, 2, 57-66.

31. Sage J.E., Gavin P.: Musculoskeletal MRI. Vet Clin North Am Small Anim Pract 2016, 46, 421-451.

32. Taylor K.W., Moore M.M., Brian J., Methratta S., Bernard S.: Wrist MR imaging in children: effect on clinical diagnosis and management. Clin Imaging 2017, 44, 61-65.

33. Terry D.W., Ramin J.E.: The navicular fat stripe: a useful roentgen feature for evaluating wrist trauma. AJR Am J Roentgenol 1975, 124, 25-28.

34. Theumann N., Favarger N., Schnyder P., Meuli R.: Wrist ligament injuries: value of post-arthrography computed tomography. Skeletal Radiol 2001, 30, 88-93.

35. Thrall D.E.: Textbook of Veterinary Diagnostic Radiology, Elsevier Health Sciences, Saint Louis 2013.

36. Tomlin J.L., Pead M.J., Langley-Hobbs S.J., Muir P.: Radial carpal bone fracture in dogs. J Am Anim Hosp Assoc 2001, 37, $173-178$.

37. Tung W.L., Zhao C., Yoshii Y., Su F.C., An K.N., Amadio P.C.: Comparative study of carpal tunnel compliance in the human, dog, rabbit, and rat. J Orthop Res 2010, 28, 652-656.

38. Turan E., Bolukbasi O.: Evaluation of possible carpal tunnel syndrome in dogs. Vet Rec 2004, 155, 122-123.

39. Turan E., Erden H.: Computed tomography and morphometry of the carpal canal in the dog. Ann Anat 2003, 185, 173-178.

40. Turan E., Ozsunar Y., Yildirim I.G.: Ultrasonographic examination of the carpal canal in dogs. J Vet Sci 2009, 10, 77-80.

41. Vaughan L.C.: Disorders of the carpus in the dog. Part I. Br Vet J $1985,141,332-341$.

42. Vaughan L.C.: Disorders of the carpus in the dog. Part II. Br Vet J 1985, 141, 435-446.

43. Willems W.F., Alberton G.M., Bishop A.T., Kremer T.: Vascularised bone grafting in a canine carpal avascular necrosis model. Clin Orthop Relat Res 2011, 496, 2832-2837.

44. Zanetti M., Saupe N., Nagy L.: Role of MR imaging in chronic wrist pain. Eur Radiol 2007, 17, 927-938. 